\title{
Isolation and Identification of Oral Bacteria and Characterization for Bacteriocin Production and Antimicrobial Sensitivity
}

\author{
Monzilur Rahman ${ }^{1}$, Md. Nahidul Islam ${ }^{1}$, Muhammad Nurul Islam ${ }^{2}$ \\ and Mohammad Shahnoor Hossain ${ }^{1}$
}

\author{
${ }^{1}$ Department of Genetic Engineering \& Biotechnology, University of Dhaka, Dhaka-1000, Bangladesh \\ ${ }^{2}$ Department of Botany, University of Dhaka, Dhaka-1000, Bangladesh
}

Received: May 10, 2015; Accepted: June 03, 2015; Published (web): June 15, 2015

\begin{abstract}
Oral bacteria play an important role in body homeostasis and the bacterial genus Streptococcus is the dominant microflora commonly found in oral bacterial community. Their ability to establish biofilm lifestyle in the oral cavity by outcompeting other bacteria has been attributed to the production of bacteriocin along with other strategies. The goal of the present study was to isolate and identify oral bacteria and characterize their ability to produce bacteriocin against other oral bacteria as well as their sensitivity to common antibiotics. We have employed deferred antagonism bacteriocin assay for bacteriocin production and disk diffusion assay for antibiotic susceptibility testing. We identified eight bacterial strains belonging to the genera Streptococcus and Enterococcus based on colony morphology, biochemical assays, 16S rDNA sequence analysis, and species-specific PCR. Antibiotic susceptibility assay indicated that some of the strains are resistant to one or more antibiotics. Our study also revealed that the isolated strains are capable of producing one or more bacteriocins against other oral bacteria. Further molecular and biochemical studies are required to understand the nature of observed bacteriocin.
\end{abstract}

Key words: Oral bacteria, bacteriocin, antibiotic, Streptococcus

\section{INTRODUCTION}

Human oral cavity is one of the most dynamic habitats for numerous bacterial species where they undergo intense interspecies competition to form multispecies biofilm structure. Various species of the genus Streptococcus, Lactobacillus, Lactococcus, Enterococcus, Staphylococcus, Corynebacterium, Veillonella and Bacteroids are the prominent bacteria commonly found in the oral cavity. ${ }^{1,2}$ Among the oral bacteria, Streptococcus and Enterococcus are two important members because they can shift their lifestyle from beneficial microflora on the surface of oral cavity and oropharynx to destructive pathogens when they gain access into the oral tissue and blood stream. Among the diseases caused by oral bacteria include dental caries, periodontitis, endocarditis, pharyngitis, pneumonia, meningitis etc. Most of the

Correspondence to: Mohammad Shahnoor Hossain Phone: 880-2-9670531/7824 (off.); 8801554593702 (Cell) Fax: 880-2-9667222

E-mail:mshahnoor@du.ac.bd

Dhaka Univ. J. Pharm. Sci. 14(1): 103-109, 2015 (June) oral Streptococcus are gram positive facultative anaerobes demonstrating highly efficient survival strategies such as the ability to adhere hard and soft tissues, cell-cell communication, biofilm formation and to cope up with the rapidly changing oral environment. ${ }^{3}$

A bacterium has to compete with other bacteria to colonize in the oral cavity. Therefore, they undergo extensive intra-species and inter-species communication which confer survival advantage in the harsh environment of oral cavity. ${ }^{3}$ Production of bacteriocin is an important mean of outcompeting other bacteria in this heterogeneous environment. Many gram positive bacteria produce bacteriocins which act like toxin against other bacteria, however, the producer strain is immune to its own bacteriocin due to immunity factor. ${ }^{4-6}$ Since the oral environment is very competitive, it is speculated that bacterial species isolated from such environment will produce inhibitory substances against other bacteria. ${ }^{7}$ 
Currently, antibiotics are the only treatment option against bacterial infection. However, the trend of antibiotic resistance has become alarming for public health and the oral bacteria are no exception. With an aim to understand the interspecies competition by bacteriocin production and the trend of antibiotic resistance among the indigenous oral bacteria, we have isolated and identified eight different oral bacterial strains and tested them for bacteriocin production and antibiotic sensitivity in the present study.

\section{MATERIALS AND METHODS}

Culture and growth. Oral bacterial sample was collected using a sterile cotton bud and dissolved into $500 \mu$ phosphate buffered saline (PBS) buffer $(0.12 \mathrm{M}$ $\left.\mathrm{NaCl}, 0.01 \mathrm{M} \mathrm{Na}_{2} \mathrm{HPO}_{4}, 5 \mathrm{mM} \mathrm{KH}_{2} \mathrm{PO}_{4}[\mathrm{pH} 7.5]\right)$. An aliquot $(100 \mu \mathrm{l})$ of bacterial suspension was spread on Mitis-Salivarius Agar (MSA) plate (Sigma, USA). MSA media shows blue colony in case of Streptococcus mitis, S. salivarius, S. mutans, S. sanguis and Entercoccus. After overnight growth in microaerophilic condition (candle jar), bacterial colonies with distinct morphology were picked from the MSA plate and subsequently cultured on Todd Hewitt medium (Sigma, USA).

Biochemical test. Isolated bacterial strains were subjected to various biochemical tests specific for oral bacteria. These tests facilitated the identification and characterization of the isolated strains. Biochemical tests used in this study were: Gram staining, catalase test, hemolysis in blood agar plate (BAP), growth in bile aesculin agar, bacitracin resistance and arginine hydrolysis, and esculin hydrolysis.

PCR amplification of 16S rDNA gene and species-specific PCR for S. mutans. Single colony of each bacterial strain was collected from solid agar plate and used for colony PCR. 614bp of 16S rDNA was amplified using the primer pair (Forward: 5'TGGAGAGTTTGATCCTGGCTCAG3' and Reverse: 5'TACCGCGGCTGCTGGCAC3') and sequenced. The sequences were subjected to homology search using BLAST against NCBI 16S ribosomal DNA sequence database. The BLAST result, along with the biochemical tests, was used to determine the species of the isolated strains. $S$. mutans strains were confirmed by the speciesspecific PCR with the primer set (Sm479F: 5'TCGCGAAAAAGATAAACAAACA-3' and Sm479R: 5'-GCCCCTTCACAGTTGGTTAG-3'). ${ }^{8}$

Phylogenetic classification. Phylogenetic relation of the isolated strains was inferred based on the 16S rDNA sequence. 10 homologous sequences with top BLAST scores were retrieved for each of the strains. ${ }^{9}$ All 16S rDNA sequences of the bacterial isolates and their homologous sequences were subjected to multiple sequence alignment using MEGA- 6 tool. ${ }^{10}$ The alignment was used to infer a neighbor-joining tree. For the construction of the tree, bootstrap method with 5000 bootstrap replication was used as the test of phylogeny. Finally, braches with bootstrap value cutoff of $75 \%$ were selected only.

Antibiotic sensitivity test. Each of the isolated strains was tested for sensitivity against various antimicrobial agents using agar disc diffusion method. ${ }^{11}$ Briefly, overnight grown bacteria was first adjusted to 0.5 McFarland turbidity standards $\left(\sim 1.0 \times 10^{8}\right.$ colony forming units or $\left.\mathrm{cfu} \mathrm{ml}^{-1}\right)$ and seeded onto Todd Hewitt agar with a sterile cotton swab. Plates were then left at room temperature for $15 \mathrm{~min}$ and wells were made using a sterile borer. The culture $(100 \mu \mathrm{l})$ was spread over the THY-agar plate. Antibiotic disks (HiMedia, India) were then impregnated on the seeded culture and incubated at microaerophilic condition for overnight. Penicillin, amikacin, amoxicillin, azithromycin, bacitracin, chloramphenicol, ciprofloxacin, metronidazole, imipenem, meropenem and tetracycline were used to assess the antibiotic sensitivity. The sensitivity to an antibiotic was expressed as one of the three categories, resistant (R), sensitive (S) or intermediate (I) based on the diameter of zone of inhibition.

Bacteriocin assay. Different bacteria produce various short peptides called bacteriocin to prevent the growth of competing bacteria. Bacteriocin production was investigated by deferred antagonism bacteriocin assay. ${ }^{12}$ Briefly, the isolated strains were stabbed into the THY agar with a sterile tooth pick and incubated at $37{ }^{\circ} \mathrm{C}$ for $24 \mathrm{~h}$ in candle jar. After 
incubation, overnight grown indicator strain was spread over the petridish seeded with the producer strains and incubated for another 24-hr. After the incubation, zone diameter around the producer strain was recorded.

\section{RESULTS AND DISCUSSION}

Isolation of bacterial strains based on colony morphology and biochemical tests. After the bacteria were grown on MSA plate, colonies with distinctive morphology were selected (Table 1). Eight colonies were selected for further study. All of the strains displayed positive gram staining reaction. Six different biochemical tests for the presumptive identification of the isolated colonies were also performed. All of the strains were catalase negative. However, they exhibited differential hemolysis pattern on blood agar and distinctive reaction pattern for bile aesculin, bacitracin resistance, arginine hydrolysis and esculin hydrolysis tests. The summary of the results for biochemical reactions has been presented in Table 2.

Table 1. Colony morphology on Mitis Salivaris agar and Gram staining of the isolated strains.

\begin{tabular}{llc}
\hline ID & Colony morphology & Gram staining \\
\hline SN1 & Undule-shaped, with a granular frosted-glass appearance & Positive \\
SN3 & Small, flat, light blue & Positive \\
SN8 & Small, flat, light blue & Positive \\
SN13 & Undule-shaped, with a granular frosted-glass appearance & Positive \\
SN15 & Glossy, grayish white, small & Positive \\
SN19 & Dark, blue-black & Positive \\
SN21 & Dark, blue-black & Positive \\
SN23 & Undule-shaped, with a granular frosted-glass appearance & Positive \\
\hline
\end{tabular}

Table 2. Biochemical tests of the isolated strains.

\begin{tabular}{lccccccc}
\hline ID & Catalase test & $\begin{array}{c}\text { Hemolysis } \\
(\mathrm{BAP})\end{array}$ & $\begin{array}{c}\text { Bile aesculin } \\
\text { agar }\end{array}$ & $\begin{array}{c}\text { Bacitracin } \\
\text { resistance }\end{array}$ & $\begin{array}{c}\text { Arginine } \\
\text { hydrolysis }\end{array}$ & $\begin{array}{c}\text { Esculin } \\
\text { hydrolysis }\end{array}$ & $\begin{array}{c}\text { Presumptive species (based on } \\
\text { sequence homology) }\end{array}$ \\
\hline SN1 & - & $\gamma$ & - & + & - & + & S. mutans \\
SN3 & - & $\alpha$ & - & - & - & - & S. oralis \\
SN8 & - & $\alpha$ & - & - & - & - & S. oralis \\
SN13 & - & $\gamma$ & - & + & - & + & S. mutans \\
SN15 & - & $\beta$ & - & - & + & - & S. pyogenes \\
SN19 & - & $\gamma$ & + & - & + & + & E. faecalis \\
SN21 & - & $\gamma$ & + & - & + & + & E. faecalis \\
SN23 & - & $\gamma$ & - & + & - & + & S. mutans \\
\hline
\end{tabular}

Table 3. Antibiotics sensitivity test of the isolated bacteria.

\begin{tabular}{llllllllllll}
\hline ID & P & AK & AMX & AZT & BAC & C & CIP & MTZ & IPM & MER & TE \\
\hline SN1 & R & S & S & S & R & S & S & S & S & S & S \\
SN3 & I & S & S & S & S & S & S & S & S & S & S \\
SN8 & S & S & S & S & S & S & S & S & S & S & S \\
SN13 & S & S & S & S & R & S & S & R & S & S & S \\
SN15 & I & S & S & S & S & S & S & S & S & S & S \\
SN19 & R & S & S & S & S & S & S & R & S & S & R \\
SN21 & R & S & S & S & S & S & S & R & S & S & S \\
SN23 & S & S & S & S & R & S & I & S & S & S & R \\
\hline
\end{tabular}

$\mathrm{P}=$ Penicillin, $\mathrm{AK}=$ Amikacin, $\mathrm{AMX}=$ Amoxicillin, $\mathrm{AZT}=$ Azithromycin, $\mathrm{BAC}=$ Bacitracin $\mathrm{C}=$ Chloramphenicol, $\mathrm{CIP}=\mathrm{Ciprofloxacin}$, $\mathrm{MTZ}=$ Metronidazole, $\mathrm{IPM}=$ Imipenem, $\mathrm{MER}=$ Meropenem, $\mathrm{TE}=$ Tetracyclin, $\mathrm{S}=$ Sensitive, $\mathrm{R}=$ Resistant, $\mathrm{I}=$ Intermediate 
Identification of bacterial isolates based on 16S rDNA sequencing and species-specific PCR. 16S rDNA of each isolate was amplified by PCR and the expected product size was found in agarose gel after electrophoresis (Figure 1a) and the PCR product was sequenced. The sequences of the purified PCR products were searched for homology using BLAST. Based on the highest BLAST score and the biochemical tests, each isolates was assigned a species name. The results of these tests are summarized in table 2. PCR with the species specific primers confirmed that three of the isolated colonies belong to the species $S$. mutans (Figure 1b).

Phylogenetic relationship among the bacterial isolates and other bacteria. A phylogenetic tree was constructed taking the isolated bacterial strains and their top BLAST hits. The tree shows their species level identification as well as their phylogenetic relation with other species (Figure 2).
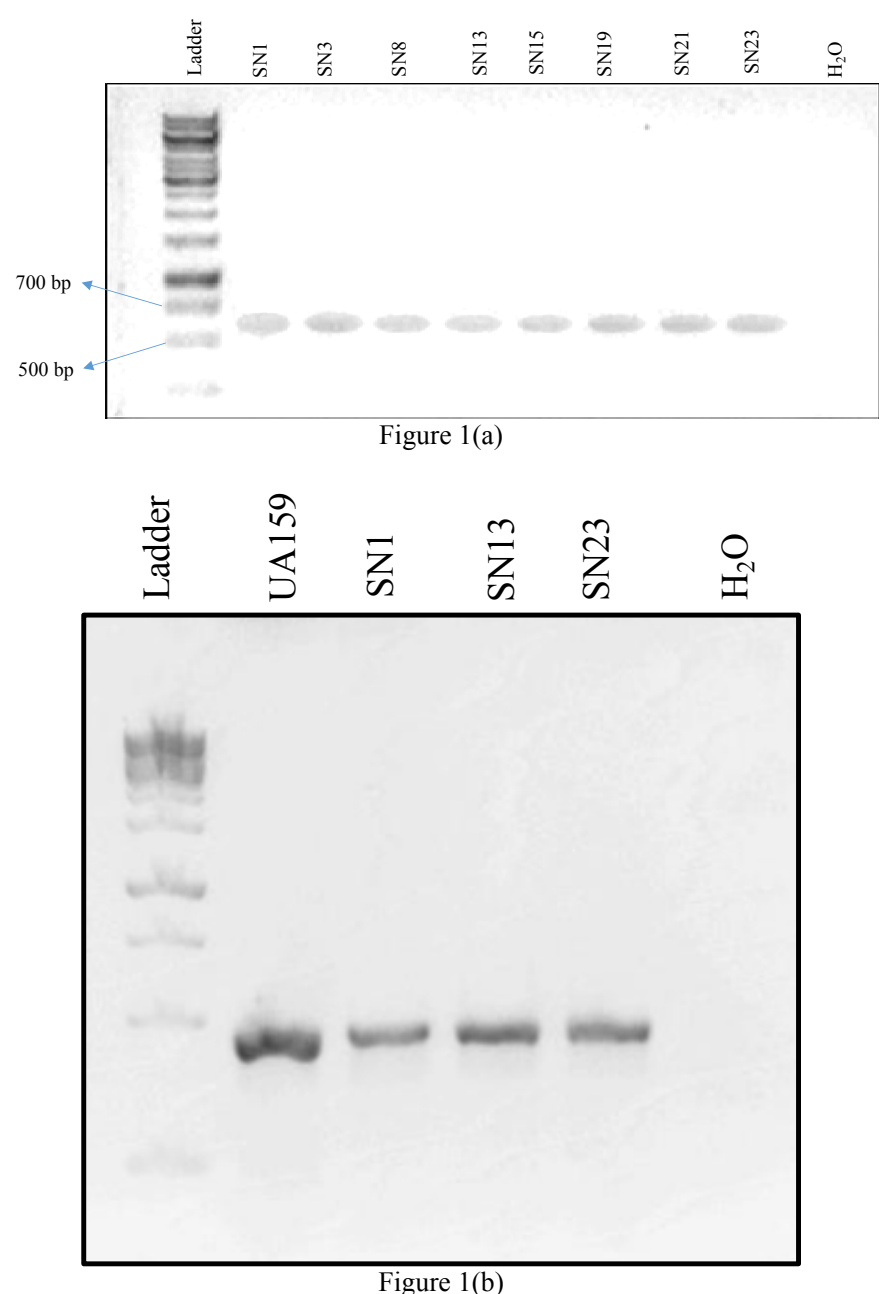

Figure 1. PCR product of partial 16S rDNA with universal primers (1a) and SM.479 with species-specific primers (1b) in agarose gel electrophoresis. Colony PCR was performed as mentioned in the materials and methods section and loaded into $1.5 \%$ agarose gel. The first lane was $1 \mathrm{Kbp}(+)$ DNA ladder. $\mathrm{H}_{2} \mathrm{O}$ was used as negative control. PCR bands for the $16 \mathrm{~S}$ rDNA were found between the position of $500 \mathrm{bp}$ band and $700 \mathrm{bp}$ of the ladder. For figure 1(b), colony PCR was performed with universal reference strain, $S$. mutans UA159 as positive control.

Antibiotic sensitivity of the isolated bacteria.

From the antibiotic sensitivity test, it is clear that the oral bacteria used in this study are sensitive to most of the antibiotics. We did not find any resistant bacteria against amikacin, amoxicillin, azithromycin, chloramphenicol, imipenem, meropenem and 
tetracycline in our study. However, some of the strains were resistant to a number of antibiotics and the resistance behavior varied in the same species (Table 3). Different patterns of resistance behavior were observed against penicillin, bacitracin, metronidazole and tetracycline. S. mutans strains
SN1, SN13 and SN23 were completely resistant to bacitracin and we observed resistance to penicillin, metronidazole and tetracycline as well. A renowned drug resistant bacterium, Enterococcus, was also found to be penicillin and metronidazole resistant in our study along with a strain resistant to tetracylcine.

Table 4. Bacteriocin production by the isolated bacteria against other bacteria.

\begin{tabular}{|c|c|c|c|c|c|c|c|c|}
\hline Producer & SN1 & $\mathrm{SN} 3$ & SN8 & SN13 & SN15 & SN19 & SN21 & SN23 \\
\hline SN3 & + & - & + & - & - & - & - & + \\
\hline SN13 & - & - & - & - & - & - & - & - \\
\hline SN15 & - & - & - & - & - & - & +++ & - \\
\hline SN21 & - & + & - & - & - & - & - & - \\
\hline SN23 & - & - & - & - & - & - & - & - \\
\hline
\end{tabular}

+ indicates moderate level of bacteriocin production, - indicates no bacteriocin production, +++ indicates increased level of bacteriocin production

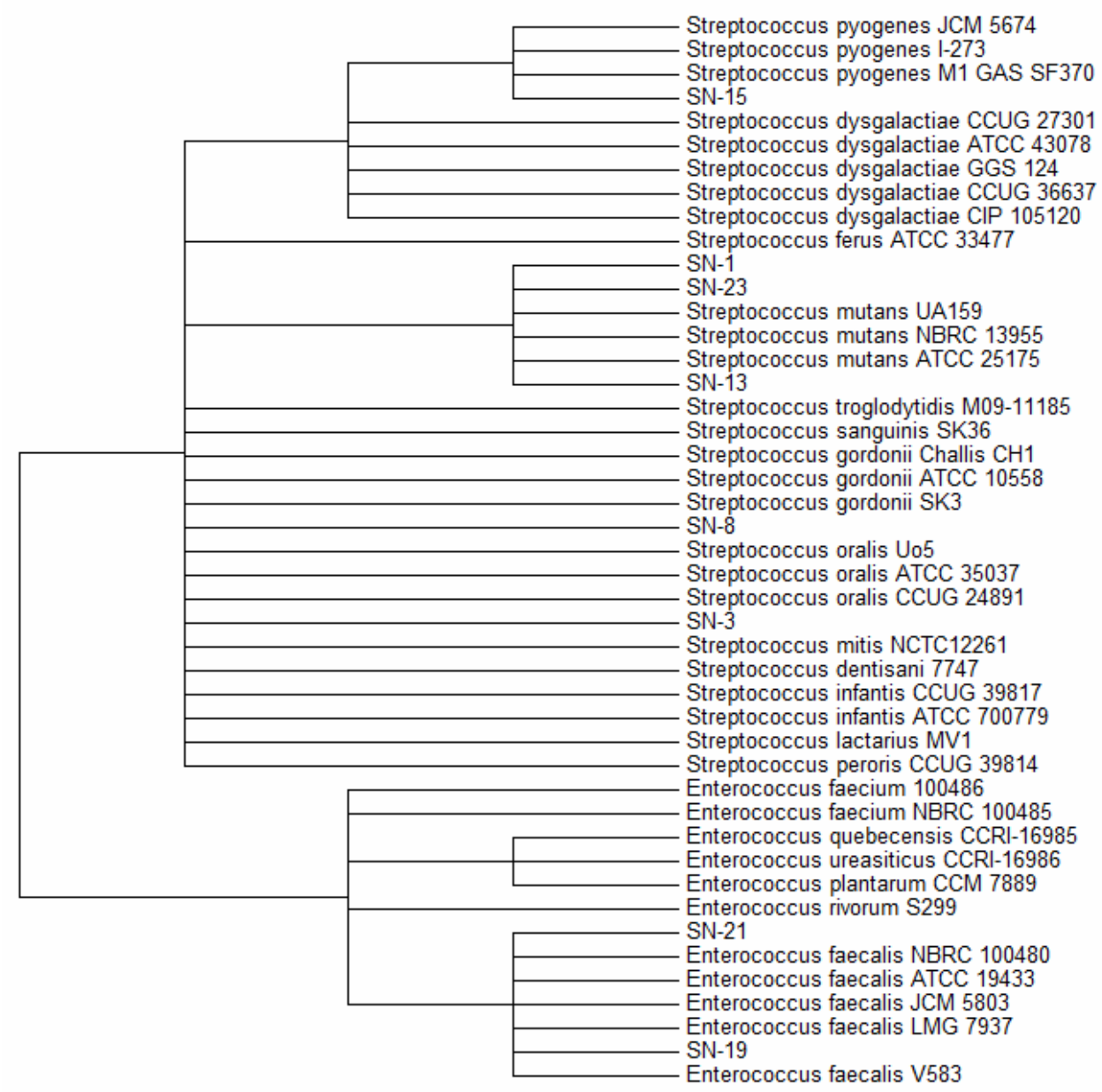

Figure 2. The phylogenetic tree was inferred using the Neighbor-Joining method. ${ }^{14}$ The optimal tree with the sum of branch length $=$ 1.34779880 is shown. The evolutionary distances were computed using the Maximum Composite Likelihood method ${ }^{15}$ and are in the units of the number of base substitutions per site. The analyses were conducted in MEGA6. ${ }^{10}$ 
Production of bacteriocin like molecules by the bacterial isolates. Deferred antagonism bacteriocin assay was performed among the isolated strains to investigate the competitive behavior against each other. Most of the bacterial isolates were shown to produce bacteriocin against other bacteria (Table 4). Bacteriocin produced by SN15 demonstrated prominent activity against the SN21. In addition, $S$. mutans SN1 produces bacteriocin against $S$. oralis (SN3), E. faecalis (SN19), and S. mutans (SN23). S. oralis $\mathrm{SN} 3$ produces bacteriocin against $S$. mutans $\mathrm{SN} 1$, S. oralis $\mathrm{SN} 8$, and S. mutans SN23. E. faecalis SN19 produces bacteriocin against $S$. oralis SN3 and S. mutans SN23. E. faecalis SN21 produces bacteriocin against $S$. oralis $\mathrm{SN} 3$.

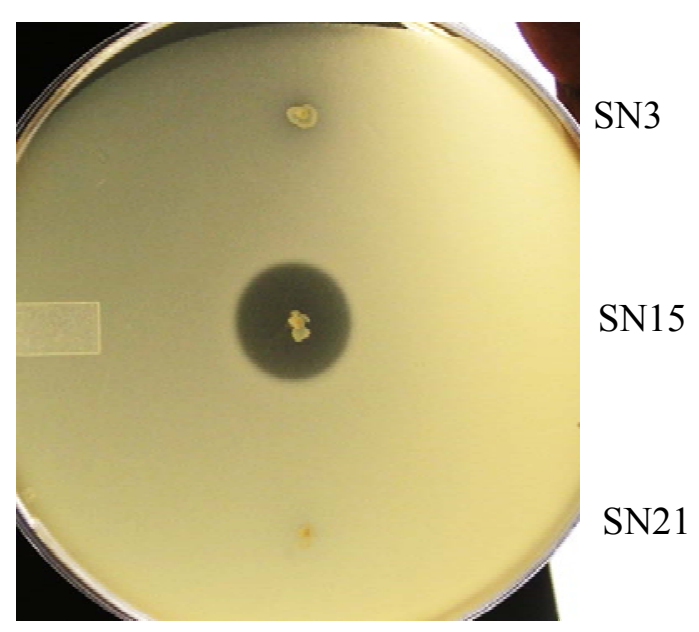

Figure 3. Production of bacteriocin by $S$. pyogenes SN15 against $E$. faecalis SN21. Isolated bacteria were stabbed into the THYagar and incubated for 24 hour. Overnight grown indicator bacteria (SN21) was overlayed as $0.4 \%$ soft agar. After overnight incubation, growth inhibition was observed. $S$. oralis $\mathrm{SN} 3$ and E. faecalis $\mathrm{SN} 21$ were used as negative control. Each of the experiment was done in triplicate and a representative plate has been presented here.

The Streptococci have developed an intimate relationship with human body over the period of time and represents the single most abundant microflora in human microbiome structure. ${ }^{13}$ They are generally present as normal microflora in the oral cavity, skin surface, upper respiratory and digestive tract. They can also cause various diseases when natural homeostasis is disturbed. It is believed that they have achieved this progess by various strategies which helped them to outcompete other bacterial population. Antimicrobial peptides or bacteriocins, synthesized by the ribosome, are considered to be an important strategic arsenal for these bacteria in the highly competitive microbial habitat. Oral Streptococcus and Enterococcus bear importance due to their ability to produce bacteriocin and resistance to various antibiotics. The goal of the present study was to isolate and identify oral bacteria and to characterize them for bacteriocin production and antimicrobial sensitivity. In this study, we have been successful to isolate and identify eight oral bacterial strains, which belong to S. pyogenes, S. mutans, S. oralis, S. pyogenes and E. faecalis. Most of the bacteria were able to produce bacteriocins against other newly isolated strain and the strain SN15 (S. pyogenes) was able to produce bacteriocin with high level of activity against SN21 (E. faecalis) (Figure 3). Antibiotic sensitivity pattern suggests that some of the strains were resistant to one or more antibiotics. Particularly, the strains belonging to the species $S$. mutans and Enterococcus were resistant to penicillin, metronidazole andtetracyline. However, all the isolated strains were sensitive to amikacin, amoxicillin, azithromycin, chloramphenicol, imipenem, meropenem and tetracyclin. Further studies are required to unravel the mechanism of antibiotic resistance among these strains.

\section{CONCLUSION}

In the present study, we were able to isolate and identify several oral bacterial strains which belonged to the species Streptococcus and Enterococcus with varying antibiotic resistance patterns. These newly isolated bacteria were able to produce bacteriocins which inhibited the growth of other bacteria. We are currently conducting experiments to purify these bacteriocins and characterize their biochemical and fermentative properties.

\section{ACKNOWLEDGEMENTS}

We thank Biotechnology Research Center for providing a grant to continue this project. We also thank Chemical Biology and DNA Research Lab, 
Faculty of Pharmacy for providing the primers for this study.

Conflict of interest: We state that there is no conflict of interest to the publication of this article.

\section{REFERENCES}

1. Rogers, A. 2008. Molecular Oral Microbiology. Norfolk, UK: Caister Academic Press.

2. Wang, Q.Q., Zhang, C.F., Chu, C.H., Zhu and X.F. 2012. Prevalence of Enterococcus faecalis in saliva and filled root canals of teeth associated with apical periodontitis. Int. J. Oral Sci. 4, 19-23.

3. Cvitkovitch, D.G., Li, Y.H. and Ellen, R.P. 2003. Quorum sensing and biofilm formation in Streptococcal infections. $J$. Clin. Invest. 112, 1626-1632.

4. Jack, R.W., Tagg, J.R. and Ray, B. 1995. Bacteriocins of gram-positive bacteria. Microbiol. Rev. 59, 171-200.

5. Tagg, J.R., Dajani, A.S. and Wannamaker, L.W. 1976. Bacteriocins of gram-positive bacteria. Bacteriol. Rev. 40, 722-756.

6. Hossain, M.S. and Biswas, I. 2012. SMU. 152 acts as an immunity protein for mutacin IV. J. Bacteriol. 194, 34863494.

7. Weerkamp, A., Bongaerts-Larik, L. and Vogels, G.D. 1977. Bacteriocins as factors in the in vitro interaction between oral streptococci in plaque. Infect. Immun. 16, 773-780.
8. Chen, Z., Saxena, D., Caufield, P.W., Ge, Y. and Wang, M. 2007. Development of species-specific primers for detection of Streptococcus mutans in mixed bacterial samples. FEMS Microbiol. Lett. 272, 154-162.

9. Zhang, Z., Schwartz, S., Wagner, L. and Miller, W. 2000. A greedy algorithm for aligning DNA sequences. J. Comput. Biol. 7, 203-214.

10. Tamura, K., Stecher, G., Peterson, D., Filipski, A. and Kumar, S. 2013. MEGA6: Molecular Evolutionary Genetics Analysis version 6.0. Mol. Biol. Evol. 30, 2725-2729.

11. Bauer, A.W., Kirby, W.M.M., Sherris, J.C. and Turck, M. 1966. Antibiotic susceptibility testing by a standard single disc method. Am. J. Clin. Pathol. 45, 493-96.

12. Hossain, M.S. and Biswas, I. 2011. Mutacins from Streptococcus mutans UA159 are active against multiple streptococcal species. Appl. Environ. Microbiol. 77, 24282434.

13. Human Microbiome Project, C. 2012. Structure, function and diversity of the healthy human microbiome. Nature 486, 207214.

14. Saitou, N. and Nei, M. 1987. The neighbor-joining method: a new method for reconstructing phylogenetic trees. Mol. Biol. Evol. 4, 406-425.

15. Tamura, K., Nei, M. and Kumar, S. 2004. Prospects for inferring very large phylogenies by using the neighborjoining method. Proc. Natl. Acad. Sci. 101, 11030-11035. 